\title{
LETTER
}

\section{Early oseltamivir therapy improves the outcome in critically ill patients with influenza: a propensity analysis}

\author{
Romain Hernu' ', Tomasz Chroboczek' , Thomas Madelaine 1,4, Jean-Sebastien Casalegno 2,3,4, Bruno Lina 2,3,4, \\ Martin Cour ${ }^{1,4}$, Laurent Argaud ${ }^{1,4,5^{*}}$ and On behalf the "Flu in Lyon ICUs"Study Group
}

๑) 2017 Springer-Verlag GmbH Germany and ESICM

Dear Editor,

Influenza affects between two and three million people worldwide each year, with complications responsible for a significant number of excess hospitalizations in intensive care units (ICUs) [1]. Since the newsworthy 2009 $\mathrm{A}(\mathrm{H} 1 \mathrm{~N} 1)$ pandemic (pdm), publications about influenza in ICUs remain scarce, with crucial outstanding issues on prognosis factors including the timing of antiviral treatments [2-5]. Here, we present a multicenter prospective study of critically ill influenza-infected patients aimed to identify prognosis factors associated with death.

This study was conducted from December 2008 to April 2013 in the 12 polyvalent ICUs from the Lyon catchment area (France). All adult patients admitted with microbiologically confirmed influenza infection were included. Following univariate comparisons, the independent contribution of patients' characteristics to in-hospital mortality was analyzed by backward stepwise multivariate analysis in a logistic regression model. Propensity score-matching was further used to compare similar patient populations receiving oseltamivir within or after 2 days of the onset of symptoms.

Over the study period, 201 patients were enrolled with the following main reasons for ICU admission: respiratory distress $(n=174,87 \%)$, shock $(n=13,6.5 \%)$, and

\footnotetext{
*Correspondence: laurent.argaud@chu-lyon.fr

${ }^{5}$ Service de Réanimation Médicale, Groupement Hospitalier Edouard Herriot, 5, place d'Arsonval, 69437 Lyon Cedex 03, France Full author information is available at the end of the article

Romain Hernu and Tomasz Chroboczek authors made equal contributions to the manuscript.

The list of co-investigators appears in the appendix at the end of the manuscript and in the ESM 2.
}

neurological failure $(n=7,3.5 \%)$. Infections were mostly caused by type A influenza virus $(n=171,85 \%)$, with $\mathrm{A}(\mathrm{H} 1 \mathrm{~N} 1) \mathrm{pdm}$ encountered in half of cases $(n=100$, $50 \%)$. Other characteristics of the patients are presented in Table 1. Only 40 (20\%) patients received antiviral treatment by oseltamivir within 2 days of the onset of symptoms. Day-28, ICU, and in-hospital mortalities were $18 \%(37 / 201), 21 \%(43 / 201)$, and $26 \%(53 / 201)$, respectively. Survivors were more likely to have received early oseltamivir therapy, with a significant trend in proportions of in-hospital mortality with increasing time from onset of symptoms to initiation of treatment $(p=0.01)$. Oseltamivir administration within 2 days of the onset of symptoms appeared as the sole independent determinant of a favorable outcome (OR 0.26 ; $95 \%$ CI $0.08-$ $0.79, p=0.02)$. After propensity score-matched analysis, oseltamivir therapy within 2 days of the onset of symptoms was associated with reduced in-hospital mortality (Supplementary Table 1).

The present study emphasizes the delay in oseltamivir administration as a major risk factor for in-hospital mortality. Influenza is a public health problem that, each year, causes both severe illness and deaths in high-risk populations [1]. With the exception of the 2009 pandemic, data on critical illnesses attributable to influenza are scarce. Thus, we designed the present study to provide current information on influenza disease in ICUs in the real situation of a specific territory. Concerning the severity of the patients' illness or the risk factors of death, our cohort is in agreement with previous studies on influenza-associated critical illness mainly drawn from 2009 pandemic studies [2,3]. Nevertheless, our study does not confirm the negative impact on patients' outcomes of the influenza $\mathrm{A}(\mathrm{H} 1 \mathrm{~N} 1) \mathrm{pdm}$ virus subtype. Importantly, as

\section{实


Table 1 Patients' characteristics according to outcome

\begin{tabular}{|c|c|c|c|c|c|}
\hline & $\begin{array}{l}\text { Total } \\
n=201\end{array}$ & $\begin{array}{l}\text { Survivors } \\
n=148\end{array}$ & $\begin{array}{l}\text { Non-survivors } \\
n=53\end{array}$ & Univariate analysis $p$ & Multivariate analysis OR $(95 \% \mathrm{CI})$ \\
\hline \multicolumn{6}{|l|}{ Clinical features } \\
\hline Age $^{a}$ (years) & $63 \pm 16$ & $62 \pm 15$ & $66 \pm 17$ & 0.088 & $1.03(1.00-1.05)^{*}$ \\
\hline Male sex & $107(53)$ & $81(55)$ & $26(49)$ & 0.477 & \\
\hline Influenza vaccination & $25(12)$ & $19(13)$ & $6(11)$ & 0.773 & \\
\hline $\mathrm{BMI}^{\mathrm{a}}\left(\mathrm{kg} \mathrm{m}^{-2}\right)$ & $27 \pm 7$ & $28 \pm 7$ & $26 \pm 7$ & 0.168 & \\
\hline No comorbidity & $19(9)$ & $14(9)$ & $5(9)$ & 0.996 & \\
\hline SAPS $\|$ & $44 \pm 17$ & $40 \pm 15$ & $54 \pm 17$ & $<0.001$ & \\
\hline \multicolumn{6}{|l|}{ Clinical course } \\
\hline $\begin{array}{l}\text { Symptom duration before ICU admission } \\
\text { (days) }\end{array}$ & $5.1 \pm 5.0$ & $5.1 \pm 4.5$ & $5.1 \pm 6.0$ & 0.931 & \\
\hline Influenza diagnosis before ICU admission & $20(10)$ & $14(9.5)$ & $6(11)$ & 0.698 & \\
\hline Days from admission to influenza diagnosis ${ }^{a}$ & $2.3 \pm 3.4$ & $2.0 \pm 2.7$ & $2.9 \pm 4.9$ & 0.112 & \\
\hline \multicolumn{6}{|l|}{ Microbiological results } \\
\hline Viral subtype & & & & 0.707 & \\
\hline Influenza A(H1N1)pdmª & $100(50)$ & $73(49)$ & $27(51)$ & - & \\
\hline Other type A & $71(35)$ & $54(37)$ & $17(32)$ & - & \\
\hline Type B & $30(15)$ & $21(14)$ & $9(17)$ & - & \\
\hline Bacterial co-infection at admission & $48(24)$ & $38(26)$ & $10(19)$ & 0.319 & \\
\hline VAP in the evolution ${ }^{a}$ & $51(25)$ & $31(21)$ & $20(38)$ & 0.016 & $1.91(0.89-4.10)$ \\
\hline \multicolumn{6}{|l|}{ Initial organ failures } \\
\hline \multicolumn{6}{|l|}{ Type } \\
\hline Respiratory & $109(54)$ & $74(50)$ & $35(66)$ & 0.044 & \\
\hline Cardiovascular & $86(43)$ & $58(39)$ & $28(53)$ & 0.003 & \\
\hline Neurological & $50(25)$ & $30(20)$ & $20(38)$ & 0.011 & \\
\hline Hematological & $24(12)$ & $8(5.4)$ & $16(30)$ & $<0.001$ & \\
\hline Renal & $21(10)$ & $10(6.8)$ & $11(21)$ & 0.004 & \\
\hline Hepatic & $2(1.0)$ & $1(0.7)$ & $1(1.9)$ & 0.459 & \\
\hline Number of failed organs & $1.5 \pm 1.2$ & $1.2 \pm 1.1$ & $2.1 \pm 1.2$ & $<0.001$ & \\
\hline SOFA score ${ }^{a}$ & $6.6 \pm 3.9$ & $5.9 \pm 3.8$ & $8.7 \pm 3.7$ & $<0.001$ & $1.19(1.08-1.30)^{*}$ \\
\hline \multicolumn{6}{|l|}{ Organ support during ICU stay } \\
\hline Mechanical ventilation & $179(89)$ & $126(85)$ & $53(100)$ & 0.003 & \\
\hline ARDS & $113(56)$ & $73(49)$ & $40(75)$ & 0.001 & \\
\hline NIV only & $36(18)$ & $31(22)$ & $5(9.4)$ & 0.061 & \\
\hline Days & $14 \pm 20$ & $12 \pm 17$ & $19 \pm 28$ & 0.084 & \\
\hline $\mathrm{ECMO}^{\mathrm{a}}$ & $8(4.0)$ & $4(2.7)$ & $4(7.5)$ & 0.211 & \\
\hline Catecholamines (days) & $4.7 \pm 8.8$ & $3.3 \pm 6.8$ & $8.2 \pm 12.4$ & 0.005 & \\
\hline RRT (days) & $3.9 \pm 12.0$ & $2.7 \pm 8.8$ & $7.3 \pm 17.9$ & 0.073 & \\
\hline \multicolumn{6}{|l|}{ Other treatments } \\
\hline Prone position ${ }^{\mathrm{a}}$ & $38(19)$ & $23(15)$ & $15(28)$ & 0.041 & \\
\hline Neuromuscular blockade & $67(33)$ & $45(30)$ & $22(42)$ & 0.141 & \\
\hline Nitric oxide & $19(9.5)$ & $11(7.4)$ & $8(15)$ & 0.101 & \\
\hline
\end{tabular}


Table 1 continued

\begin{tabular}{|c|c|c|c|c|c|}
\hline & $\begin{array}{l}\text { Total } \\
n=201\end{array}$ & $\begin{array}{l}\text { Survivors } \\
n=148\end{array}$ & $\begin{array}{l}\text { Non-survivors } \\
n=53\end{array}$ & Univariate analysis $p$ & Multivariate analysis OR $(95 \% \mathrm{CI})$ \\
\hline Oseltamivir & $146(73)$ & $109(74)$ & $37(70)$ & 0.591 & \\
\hline Administration before ICU admission & $17(8.5)$ & $13(8.7)$ & $4(7.5)$ & 0.999 & \\
\hline Dose (mg/day) & $201 \pm 84$ & $197 \pm 82$ & $215 \pm 98$ & 0.257 & \\
\hline$\leq 2$ days after onset of symptoms ${ }^{a}$ & $40(20)$ & $36(24)$ & $4(7.5)$ & 0.009 & $0.26(0.08-0.79)^{*}$ \\
\hline Days from onset of symptoms to initiation & $4.9 \pm 3.5$ & $4.6 \pm 3.6$ & $5.7 \pm 3.1$ & 0.086 & \\
\hline Steroids ${ }^{a}$ & $91(45)$ & $63(43)$ & $28(53)$ & 0.288 & \\
\hline
\end{tabular}

Data are number (\%) or mean \pm standard deviation, as appropriate

OR odds ratio, Cl confidence interval, BMI Body Mass Index, ICU intensive care units, VAP Ventilator-Associated Pneumonia, ARDS Acute Respiratory Distress Syndrome, SOFA Sepsis-Related Organ Failure Assessment, NIV non-invasive ventilation, ECMO extra-corporeal membrane oxygenation, RRT renal replacement therapy

${ }^{*} p<0.05$

a Variables included in the backward stepwise logistic regression

confirmed by propensity analysis, oseltamivir administration was associated with better outcomes when administrated within 2 days of the onset of symptoms. This is the key message of our work, consistent with recent studies in non-severe forms of the disease, including meta-analysis of randomized clinical trials [4]. Indeed, the recent literature highlights the efficacy of oseltamivir to reduce the duration of symptoms, respiratory tract complications, and hospital admittance $[4,5]$. Our results extend to the ICU setting the relationship between the delay of oseltamivir administration and the effectiveness of the treatment in patients with either $\mathrm{A}(\mathrm{H} 1 \mathrm{~N} 1) \mathrm{pdm}$ or other influenza virus subtypes.

In conclusion, this real-life study emphasizes oseltamivir efficacy on in-hospital outcome when administrated within 2 days of the onset of symptoms. Even if physicians' awareness of the influenza disease has been undeniably enhanced since the last pandemic, many efforts are still required to improve influenza-infected patient management in ICUs, including early oseltamivir administration.

\section{Electronic supplementary material}

The online version of this article (doi:10.1007/s00134-017-4953-3) contains supplementary material, which is available to authorized users.

\section{Author details \\ 1 Service de Réanimation Médicale, Hospices Civils de Lyon, Groupement Hos- pitalier Edouard Herriot, 69003 Lyon, France. ${ }^{2}$ Laboratoire de Virologie Est, CNR des virus influenza (sud France), Hospices Civils de Lyon, Centre de Biologie et de Pathologie Est, 69500 Bron, France. ${ }^{3}$ Centre International de Recherche en Infectiologie, INSERM U1111, CNRS, UMR 5308, Ecole Normale Supérieure de Lyon, Lyon, France. ${ }^{4}$ Faculté de médecine Lyon-Est, Université de Lyon, Université Lyon 1, 69008 Lyon, France. ${ }^{5}$ Service de Réanimation Médicale, Groupement Hospitalier Edouard Herriot, 5, place d'Arsonval, 69437 Lyon Cedex 03, France.}

\section{Acknowledgements}

Co-investigators: Members of the "Flu in Lyon ICUs"Study Group: Hospices Civils de Lyon, Hôpital de la Croix-Rousse, Lyon, France: F. Aubrun, F. Bayle, G. Bourdin, C. Guérin, L. Josset; Hospices Civils de Lyon, Hôpital Edouard Herriot, Lyon, France: B. Allaouchiche, C. Augier, T. Baudry, B. Delwarde, L. Jacquet, D. Robert, J.M. Robert, M. Simon; Hospices Civils de Lyon, Hôpital Lyon-Sud,
Lyon, France: J. Bohé; Hôpital Desgenettes, Lyon, France: M. Puidupin, J. Turc; Hôpital Privé Saint-Joseph Saint-Luc, Lyon, France: J. Manchon; Hôpital Privé Tonkin, Lyon, France: L. Liron.

\section{Compliance with ethical standards}

\section{Funding}

None.

\section{Conflicts of interest}

The authors declare they have no conflict of interest.

\section{Ethical approval}

All procedures performed in studies involving human participants were in accordance with the ethical standards of our institutional research committee and with the 1964 Declaration of Helsinki and its later amendments. For this type of study formal consent was not required.

Accepted: 30 September 2017

Published online: 12 October 2017

\section{References}

1. Thomson WW, Shay DK, Weintruab E, Brammer L, Bridges CB, Cox NJ, Fukuda K (2004) Influenza-Associated Hospitalizations in the United States. JAMA 292:1333-1340

2. Kumar A, Zarychanski R, Pinto R, Cook DJ, Marshall J, Lacroix J, Stelfox T, Bagshaw S, Choong K, Lamontagne F, Turgeon AF, Lapinsky S, Ahern SP, Smith O, Siddiqui F, Jouvet P, Khwaja K, McIntyre L, Menon K, Hutchison J, Hornstein D, Joffe A, Lauzier F, Singh J, Karachi T, Wiebe K, Olafson K, Ramsey C, Sharma S, Dodek P, Meade M, Hall R, Fowler RA, Canadian Critical Care Trials Group H1N1 Collaborative (2009) Critically ill patients with 2009 influenza A(H1N1) infection in Canada. JAMA 302:1872-1879

3. Influenza Investigators ANZIC, Webb SA, Pettilä V, Seppelt I, Bellomo R, Bailey M, Cooper DJ, Cretikos M, Davies AR, Finfer S, Harrigan PW, Hart GK, Howe B, Iredell JR, McArthur C, Mitchell I, Morrison S, Nichol AD, Paterson DL, Peake S, Richards B, Stephens D, Turner A, Yung M (2009) Critical care services and 2009 H1N1 influenza in Australia and New Zealand. N Engl J Med 361:1925-1934

4. Dobson J, Whitley RJ, Pocock S, Monto AS (2015) Oseltamivir treatment for influenza in adults: a meta-analysis of randomised controlled trials. Lancet 385:1729-1737

5. Muthuri SG, Venkatesan S, Myles PR, Leonardi-Bee J, Al Khuwaitir TS, Al Mamun A, Anovadiya AP, Azziz-Baumgartner E, Báez C, Bassetti M, Beovic B, Bertisch B, Bonmarin I, Booy R, Borja-Aburto VH, Burgmann H, Cao B, Carratala J, Denholm JT, Dominguez SR, Duarte PA, Dubnov-Raz G, Echavarria M, Fanella S, Gao Z, Gérardin P, Giannella M, Gubbels S, 
Herberg J, Iglesias AL, Hoger PH, Hu X, Islam QT, Jiménez MF, Kandeel A, Keijzers G, Khalili H, Knight M, Kudo K, Kusznierz G, Kuzman I, Kwan AM, Amine IL, Langenegger E, Lankarani KB, Leo YS, Linko R, Liu P, Madanat F, Mayo-Montero E, McGeer A, Memish Z, Metan G, Mickiene A, Mikić D, Mohn KG, Moradi A, Nymadawa P, Oliva ME, Ozkan M, Parekh D, Paul M, Polack FP, Rath BA, Rodríguez AH, Sarrouf EB, Seale AC, Sertogullarindan B, Siqueira MM, Skret-Magierlo J, Stephan F, Talarek E, Tang JW, To KK, Torres A, Törün SH, Tran D, Uyeki TM, Van Zwol A, Vaudry W, Vidmar T, Yokota
RT, Zarogoulidis P, PRIDE Consortium Investigators, N-Guyen-Van-Tam JS (2014) Effectiveness of neuraminidase inhibitors in reducing mortality in patients admitted to hospital with influenza A H1N1pdm09 virus infection: a meta-analysis of individual participant data. Lancet Respir Med 2:395-404 